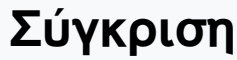

Tóp. 26 (2016)

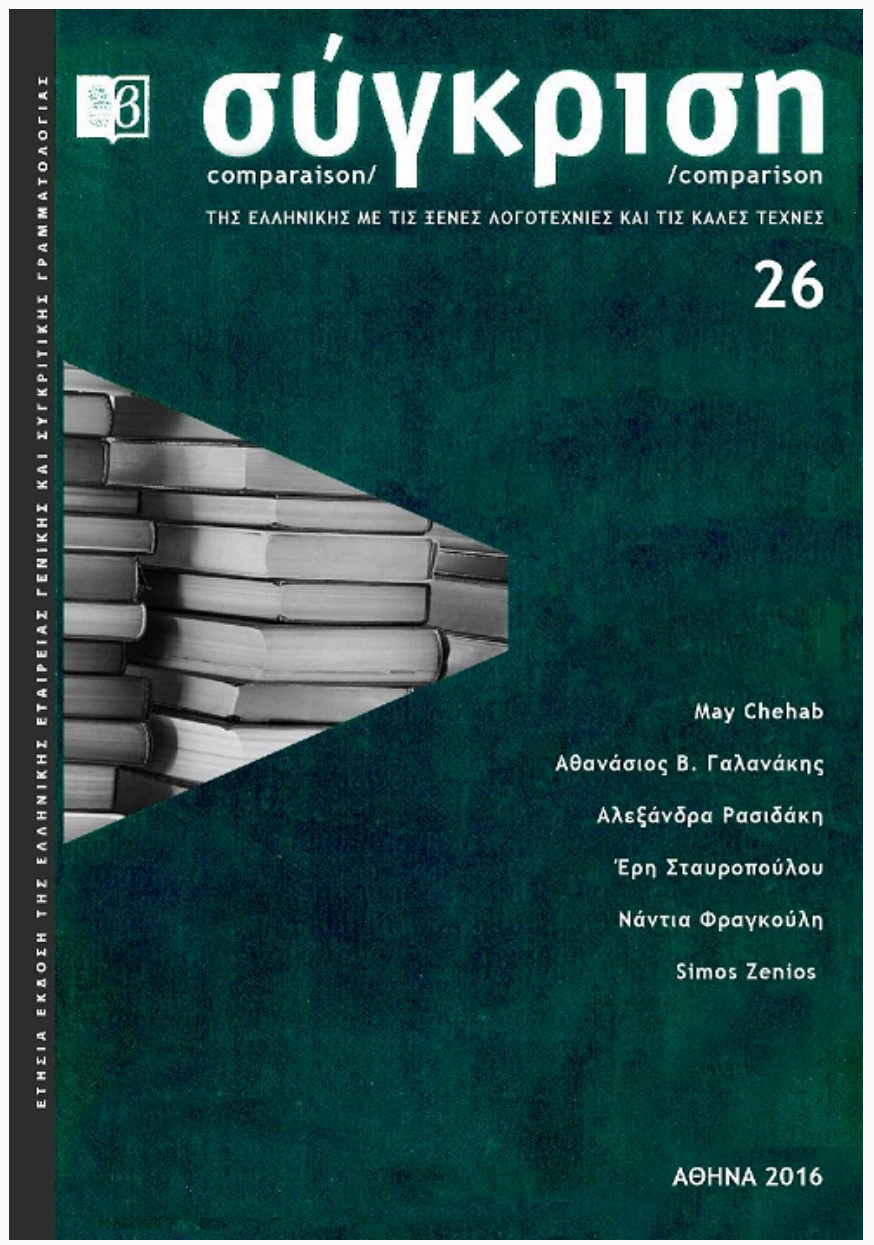

Hommage à Yourcenar : une réflexion écologique d'avant-garde

\section{May Chehab}

doi: $10.12681 /$ comparison.16006

\section{Copyright $\odot$ 2018, May Chehab}

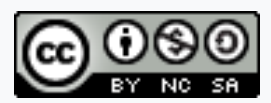

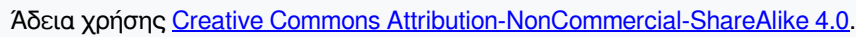

\section{Bıß入ıорачıкń avaфopá:}

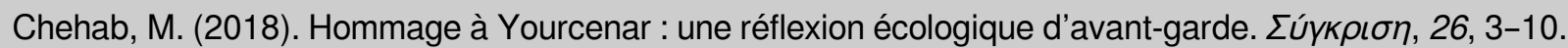
https://doi.org/10.12681/comparison.16006 


\title{
Hommage à Yourcenar : une réflexion écologique d'avant-garde
}

\author{
May Chehab \\ Université de Chypre
}

\section{Introduction}

En 2017, soit 30 ans après la disparition de Marguerite Yourcenar (1903-1987), son œuvre demeure extraordinairement pertinente: autant le très moderne examen de conscience des Mémoires d'Hadrien sur les rapports entre morale personnelle et morale publique, que l'introspection humaniste du Zénon de L'OEuvre au Noir, n'ont rien perdu de leur actualité. Participant de la même interrogation, à la fois éthique et politique, le thème qui va particulièrement retenir notre attention dans la présente étude est la nature du souci écologique de Yourcenar. ${ }^{1}$ Pour la défense d'un environnement compris de manière moins anthropocentriste que globale, tous les supports lui sont bons: si le souci du monde émaille son œuvre littéraire, il est manifeste dans sa correspondance, ses discours, ses contributions à des ouvrages collectifs, ses entretiens accordés à la presse écrite et audio-visuelle, et dans un activisme qui va au-delà d'un environnementalisme purement intellectuel. En un mot, la question écologique préoccupe Yourcenar au plus haut point, depuis son enfance au Mont-Noir jusqu'à l'île des Monts Déserts, jusque la dernière année de sa vie: en 1987, l'académicienne prononce à Québec l'allocution d'ouverture de la Ve Conférence internationale de Droit constitutionnel, entièrement consacrée à l'environnement. Et jusque même au-delà de sa vie : le « Marguerite Yourcenar Trust », qui gère post mortem les droits issus de son œuvre, compte un nombre aussi impressionnant qu'éloquent d'associations ou d'organisations non gouvernementales bénéficiaires de ses dons.

\section{Surpeuplement}

Dans son œuvre, ${ }^{2}$ d'abord, le souci de soi, de la «citadelle intérieure » des stoïciens, ne se conçoit pas sans le souci des autres : autres hommes ou femmes que soi, et autres êtres vivants sur terre. Nombreuses sont dans l'œuvre de Marguerite Yourcenar les attaques contre la légèreté de l'homme, cet « apprentisorcier» comme elle l'appelle, face aux richesses de la terre. Également problématique est l'impérialisme humain, qui ne songe pas aux désastres consécutifs au surpeuplement de la planète.

Le surpeuplement ou la surpopulation se définit comme un excès de population soit par rapport à des ressources disponibles, soit par rapport à des normes données (logement, classes). Cette notion ne peut donc être que relative et n'a que très peu de sens à l'échelle d'un pays ou d'une région. Mais à l'échelle de la Terre, à mesure que l'on s'éloigne des particularismes pour accéder aux grands nombres, le quantifiable perd son caractère relatif. En un anachronisme propre à l'écriture yourcenarienne, Zénon, le médecin et philosophe de la

\footnotetext{
${ }^{1}$ Nous nous pencherons ailleurs sur la tout aussi ardente défense des droits civiques de la part de Yourcenar (libertés publiques, droits des réfugiés, des minorités, droit à l'avortement, etc.).

2 Sauf mention contraire, les renvois se font aux deux volumes de ses OEuvres complètes, tomes I (CEuvres romanesques/OR, 1982) et II (Essais et Mémoires/EM,1991).
} 
renaissante OEuvre au Noir, se fait le défenseur moderne de la planète surpeuplée :

- Et qu'es-tu toi-même, cria Zénon pris de furie [il s'adresse au tisserand Colas Gheel], sinon une machine mal graissée qu'on use, qu'on jette au rebut, et qui par malheur en engendre d'autres? (L'CEuvre au Noir, I, 593).

Le surpeuplement fonctionne chez Yourcenar comme coefficient amplificateur des tourments des hommes :

La brutalité, l'avidité, l'indifférence aux maux d'autrui, la folie et la bêtise régnaient plus que jamais sur le monde, multipliées par la prolifération de l'espèce humaine. (Souvenirs pieux, II, 738)

Nous pourrions multiplier les exemples tirés de l'œuvre, très nombreux, pour illustrer la défense de la nature vivante et minérale. Mais la littérature critique, à laquelle nous renvoyons, est abondante quant à la place de l'écologie dans l'œuvre yourcenarienne. Nous proposons plutôt ici d'examiner son prolongement hors de l'œuvre, dans des discours et conférences, et dans un activisme délégué à divers organismes par voie de dons. Certes, comme on verra, l'inspiration environnementale yourcenarienne a été essentiellement infléchie par son rapport personnel au monde naturel, depuis son enfance au Mont-Noir jusqu'à l'île des Monts Déserts. Elle a aussi été influencée par ses lectures occidentales (Henry David Thoreau, Rachel Carson) ou extrême-orientales (bouddhisme). Sur ce fond affectif et culturel vient se greffer, dans les années cinquante où commence à s'attester l'activisme yourcenarien, une écologie combattante qui est alors fondamentalement une manifestation socioculturelle anglo-saxonne.

\section{Discours et conférences}

Accordés à la presse écrite ou audiovisuelle, ${ }^{3}$ ou prononcés en diverses circonstances comme la Ve Conférence internationale de Droit constitutionnel déjà évoquée, les conférences et discours prononcés par Yourcenar se complètent et condensent les opinions exprimées dans son œuvre littéraire, ${ }^{4}$ pour construire une pensée écologique apparentée aux préoccupations modernes les plus creusées. ${ }^{5}$

Leur ton est net et leur portée multiple: la ferme condamnation par Marguerite Yourcenar, en 1987 (Sauver..., 32), de l'appellation «Terre des Hommes » (nous soulignons) d'une importante organisation non gouvernementale place immédiatement son discours écologique sur une triple

\footnotetext{
${ }^{3}$ Notamment Yourcenar, L'Écologie.

4 Tels le Discours de Réception à l'Académie française de Marguerite Yourcenar, 1981 et le Discours d'inauguration de la «Fondation Marguerite Yourcenar » prononcé à Bailleul le 11 mars 1982, repris le 30 septembre 1987 lors de la Ve Conférence internationale de droit constitutionnel (Québec) et publié à titre posthume sous le titre «...Si nous voulons encore essayer de sauver la terre » (21-23), dorénavant Sauver... .

5 Cette réflexion développe une Introduction de Chehab 69-71. On se reportera également avec fruit à l'étude de Bonali Fiquet45-54.
} 
scène : 6 philosophique, par la place de l'homme dans l'univers ; politique, par la pragmatique même du discours ; éthique, par les principes moraux convoqués. Car cette dénomination de terre des hommes, selon Yourcenar " extrêmement dangereuse» (Sauver..., 32), entérine par son génitif toute la tradition anthropocentriste et utilitariste de mainmise sur la nature pour laquelle la satisfaction des besoins humains reste le dessein essentiel. La terre est donc


anthropisées, c'est-à-dire habitées ou exploitées par l'homme, à qui elles appartiennent, ce que Marguerite Yourcenar censure très fermement. Si, comme l'académicienne en est à juste titre convaincue, l'homme s'y croit le « prédateurroi » (Archives du Nord, II,957), c'est en usurpant de pouvoirs qui, « de quelque manière qu'on les évalue, constituent une anomalie dans l'ensemble des choses » (ibid.).

Le discours écologiste yourcenarien ne se démarque guère, à première vue, de l'environnementalisme moderne: il naît principalement du spectacle de la dégradation de la nature par l'homme. Pour la femme de lettres qu'est Yourcenar, le constat porte d'abord sur des dégâts anciens dont témoignent les œuvres littéraires (lyriques grecs, poètes français...). Il porte ensuite sur les dégâts de nos temps industrialisés, recensant toutes les ingérences malvenues de l'apprenti sorcier qu'est devenu l'homme moderne (barrage d'Assouan, Bhopal, etc.) :

On a si vite, de plus, oublié. Les événements se succèdent si rapidement que, au lieu que l'intérêt cumule, les différentes émotions semblent s'annuler les unes les autres. On a quasi oublié Bhopal, ou bien Bhopal est devenue simplement une discussion juridique qui n'intéresse que peu de gens. [...] Et on oublie, en particulier, que ces événements, qu'on croit finis après avoir lu un ou deux journaux, se poursuivent pourtant. Par exemple, que les effets de la pollution du Rhin à Bâle se sont continués sur les rives du Zwin et de la frontière hollandaise. On a oublié Tchernobyl. Même à Athènes [...], les Cariatides s'émiettent sous leurs portiques, grâce, si l'on peut dire, aux quatorze raffineries de pétrole installées sur la rive d'Éleusis et toutes installées il y a à peine une quinzaine ou une vingtaine d'années sous le règne des colonels. Nous avons à peu près oublié le règne des colonels, mais les effets sont là. (Sauver..., 29-30)

En fustigeant par l'anaphore («on a oublié ») l'un des grands dangers encourus par la conscience morale d'aujourd'hui, à savoir sa désensibilisation du fait d'une urgence médiatique constante, ${ }^{8}$ Yourcenar verse une pièce de plus au procès instruit par Kant contre la paresse intellectuelle et morale. ${ }^{9}$

\footnotetext{
${ }^{6}$ Terre des Hommes est une organisation non gouvernementale fondée en 1960 à Lausanne pour l'amélioration de la vie quotidienne des groupes d'enfants les plus vulnérables. L'anathème yourcenarien frappe la dénomination de l'ONG et non ses objectifs.

7 De genre non encore complètement fixé par l'usage, le mot, qui partage son étymologie avec

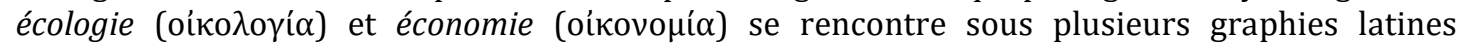
différentes.

${ }^{8}$ Voir à ce sujet Vitalis et al.

${ }^{9}$ «La paresse et la lâcheté sont les causes qui expliquent qu'un si grand nombre d'hommes, après que la nature les a affranchis depuis longtemps d'une (de toute) direction étrangère, reste
} 


\section{Activisme}

Pour l'aristocrate Marguerite de Crayencour qui, avec l'anagramme de son pseudonyme littéraire puis légal, a volontairement laissé choir sa particule, le combat est né très tôt. Il prend forme au temps d'une enfance matériellement choyée, mais caractérisée par l'étude, la sensibilité et la solitude. Là se développe, chez la petite Marguerite orpheline depuis la mort de sa mère en couches, le sentiment d'une solidarité intime avec le monde animal. ${ }^{10}$ Cette sympathie spontanée sera d'abord à la base de son végétarisme, dont on retrouve la trace dans le dernier volume de sa trilogie du Labyrinthe du Monde, Quoi ? L'éternité :

J'eus une chèvre blanche dont Michel [le père de Marguerite] dora luimême les cornes, bête mythologique avant que je sache ce qu'était la mythologie. [...] J'eus un gros mouton tout blanc qu'on savonnait chaque samedi dans la cuve de la buanderie [...]. Animal encore, et en même temps récipient sacré, ustensile magique, le premier jouet dont je me souviens: une vache en fer-blanc ou en tôle, entièrement tendue d'une vraie peau de vache, et dont la tête tournait de droite à gauche en faisant meuh. [...] J'avais repoussé dès l'époque du sevrage tout élément carné ; mon père respecta ce refus. On me nourrit bien, mais autrement. Vers l'âge de dix ans, j'appris à manger de la viande « pour faire comme tout le monde » [...] Quarante ans plus tard, révoltée par les carnages de bêtes, je repris le chemin suivi dans l'enfance. (Quoi ? L'éternité, II, 1328-1329)

De fait, Yourcenar n'a eu de cesse de dénoncer les atrocités faites aux animaux dont, comme Zénon, il lui déplaisait de « digérer [l]es agonies » (L'OEuvre au Noir, I, 703). ${ }^{11}$ Mais il s'agit là bien plus que de sympathie. L'enfant reconnaît instinctivement que, comme les créatures que l'homme nomme "bêtes ", elle appartient au même règne du vivant. L'animal est un frère ou une sœur, un alter ego : « les créatures vivantes, nos frères » (Sauver..., 30), dira-t-elle au crépuscule de sa vie:

J'eus une ânesse qui s'appelait Martine, comme tant d'ânesses, et son ânon prénommé Printemps qui trônait à son côté. Je me souviens moins de les avoir montés que d'avoir embrassé chaque jour la mère et le petit. Mais, en fait d'âne, j'avais déjà eu, plus petite encore, un amour à la Titania pour un grison qui promenait les enfants dans une île du bois de la Cambre à Bruxelles.12 [...] j'aimais tant cet âne que j'éclatai en sanglots quand, après trois tours d'île, il fallut le quitter. [...] Je rentrai au Mont-Noir assombrie par ce premier chagrin d'amour. (Quoi? L'éternité,II, 1329)

\footnotetext{
cependant volontiers, leur vie durant, mineurs, et qu'il soit facile à d'autres de se poser en tuteur des premiers » (Kant 363-493).

10 Parmi d'autres études, on se reportera notamment à : Bulletin du Cidmy; Evano 211-218 ; Torres Mariño 199-210 ; Fort 199-214.

11 Repris dans un entretien : Yourcenar, Portrait d'une Voix, 148.

${ }^{12}$ Suite à un enchantement, Titania, reine des elfes dans Le Songe d'une nuit d'été de Shakespeare, tombe amoureuse du premier venu à son réveil, qui sera Nick Bottom, également victime d'un sort qui l'a métamorphosé en âne.
} 
L'empathie enfantine se transformera en conviction d'adulte. Dès les années 1950, qui la trouvent installée aux États-Unis sur une île isolée du Maine, Yourcenar va offrir son soutien actif aux associations d'aide aux animaux. En premier lieu, à celles vouées à la défense des ânes ou chevaux. Yourcenar cotise ainsi à l'American Fondouk, association à but non lucratif (comme elles le sont toutes) fondée en 1927 pour améliorer les conditions de vie désastreuses des ânes de Fez (Maroc) -et par voie de conséquence celles de leurs propriétaires. Elle soutient aussi l'American Horse Protection Association, vouée à la protection des chevaux sauvages et domestiques.

D'un âne singulier, Yourcenar s'élève par induction empathique à tous les ânes. Et de tous les ânes à tous les animaux. Ce mouvement de l'esprit n'est pas nouveau. Dans le deuxième volume de la trilogie autobiographie Le Labyrinthe $d u$ Monde, Yourcenar précise :

La famille proprement dite m'intéresse moins que la gens, la gens moins que le groupe, l'ensemble des êtres (Archives du Nord, II, 974).

L'induction de l'individu à l'espèce est le moyen par lequel elle cherche sa place -qui est petite, dit-elle- dans le monde: elle s'accomplit dans une recherche des origines perdues, où le temps de l'espèce se substitue à celui de l'individu. La grande chaîne des êtres où se dilue l'amont de Marguerite comprendra tout aussi bien l'homme que la bête. ${ }^{13}$ Par son opposition à toute forme de discrimination entre les espèces, Yourcenar pourrait être reliée à l'antispécisme de la première heure. ${ }^{14}$ Dans une lettre de décembre 1977 , elle conclut en effet :

Enfin, égalité totale de tous les êtres humains sans distinction de sexe et de couleur. Et pourquoi pas égalité de tous les êtres sans distinction d'espèce? («Lettre à Odette Schwartz du 31 décembre 1977 », Lettres 581).

C'est donc à la grande chaîne de tous les êtres confondus que Yourcenar va contribuer. On retiendra d'abord, parmi la pléthore d'associations inscrites sur la liste du Yourcenar Trust, 15 la Société américaine de protection des animaux (The Humane Society of the United States) qui défend, comme Yourcenar, l'idée que les animaux ont une valeur intrinsèque et sont dotés de conscience. Cette société, créée au Canada en 1969, s'emploie à secourir et à protéger les animaux dans le monde entier par l'adoption de politiques, de législations et par l'éducation, et accorde autant d'importance au bien-être animal qu'à la conservation des espèces. Ses nombreux combats prennent des formes diverses : lutte contre la cruauté envers les animaux, contre les élevages de chiens, les élevages en usine,

\footnotetext{
${ }^{13}$ La théorie de l'émergence n'est pas nouvelle : elle a des racines épicuriennes. Plus près de nous, le Rêve de d'Alembert décrit la genèse de d'Alembert lui-même qui commence à un état préembryonnaire. Depuis Locke, la cartographie du moi déborde les contours fermes de l'individu et se dilue dans la grande chaîne des êtres.

14 Théorie en éthique animale datant des années 1970, l'antispécisme défend la thèse selon laquelle l'espèce à laquelle appartient un animal ne saurait être un critère pertinent pour décider de la manière dont on doit le traiter et de la considération morale qu'on doit lui accorder.

15 Citons seulement ici La Société nationale Audubon (ou National Audubon Society), le Stanwood Wildlife Sanctuary, l' United Action for Animals, l' African Fund for Endangered Wildlife, l' African Wildlife Foundation, l' Animal Rescue League of Boston, la Bangor Humane Society, les Friends of the Sea Otter, The Fund for Animals, The Cousteau Society.
} 
les abattages de phoques, les chasses en captivité et le commerce de la faune ; elle aide aussi La Société internationale pour la protection des animaux (International Society for Animal Rights) dont la mission exclusive est d'utiliser l'éducation et la législation en faveur des animaux. Incorporant elle aussi dans ses statuts le principe moral du droit des animaux, cette société a de fait été à la source de la première mention de l'expression «droits des animaux » par une cour fédérale aux États-Unis.

Contrairement à ce que l'on pourrait croire, pour l'anti-idéaliste Yourcenar, l'activisme en faveur du monde animal puise moins dans le sentimentalisme de son enfance que dans une conviction devenue philosophique, à la fois bouddhiste et post-humaniste. ${ }^{16}$ L'homme et l'animal, encore simultanément présents dans les chimères de la mythologie grecque, ont été séparés par la grande tradition occidentale platonicienne, chrétienne et cartésienne. Sur la base de cette séparation - «Une civilisation à cloisons étanches", dira Yourcenar dans Le Figaro (16.2.1972) - s'est érigée la disjonction entre, d'un côté, l'animalité de la bête, le mépris du corps, le matérialisme de la nature, et de l'autre l'exception humaine, la glorification de la parole et de l'esprit, la spiritualité de l'humanité, l'abstraction et l'idéalisme. ${ }^{17}$ En 1981, Marguerite Yourcenar résume sa pensée à l'occasion d'une conférence prononcée à Lisbonne, intitulée "Qui sait si l'âme du fils d'Adam va en haut, et si l'âme des bêtes va en bas?» (titre tiré de l'EcclésiasteIII, 21). ${ }^{18}$ Elle y accuse l'interprétation chrétienne de "l'injonction biblique de Jéhovah à Adam avant la faute, lui montrant le peuple des animaux, les lui faisant nommer, et l'en déclarant maître et seigneur » (II, 373-374). Elle y accuse encore «l'humanisme, au sens récent et abusif du mot, qui prétend n'accorder d'intérêt qu'aux réalisations humaines » (II, 373-374)

\section{Environnement}

La perspective va encore s'élargir : de l'ânon aux espèces, et puis du vivant au minéral. C'est vers 1941 que Yourcenar commence à se placer dans la perspective de l'universel. Dans un texte prophétique, elle prévoit telle Cassandre :

Des centaines d'espèces animales qui avaient réussi à survivre depuis la jeunesse du monde seront en quelques années anéanties pour des motifs de lucre et de brutalité ; l'homme arrachera ses propres poumons, les grandes forêts vertes. L'eau, l'air, et la protectrice couche d'ozone, prodiges quasi uniques qui ont permis la vie sur la terre, seront souillés et gaspillés (Archives du Nord, II, 1180).

Elle-même qualifie ce pas comme un «passage de l'archéologie à la géologie, de la méditation sur l'homme à la méditation sur la terre » (Théâtre I, 146). Elle s'y réfère encore dans son Discours de réception prononcé à l'Académie française :

\footnotetext{
${ }^{16 E t}$ ante-derridienne. L'animal autobiographique (1999) et la publication posthume au titre parodiquement cartésien L'animal que donc je suis (2006) de Jacques Derrida participent de la même dénonciation de la réification de l'animal par l'homme.

17 Pour une lecture plus récente sur la question, on se reportera à Schaeffer.

18 La conférence est d'abord publiée en janvier 1981 dans Raiz e Utopia, puis reprise sous le titre «Qui sait si l'âme des bêtes va en bas ? » dans Le Temps, ce grand sculpteur XI (II, 370-376).
} 
Ces années furent celles où, cherchant dans le passé un modèle resté imitable, j'imaginais comme encore possible l'existence d'un homme capable de "stabiliser la terre», donc d'une intelligence humaine portée à son plus haut point de lucidité et d'efficacité. Mais c'est aussi le moment où je commençais à fréquenter, avec une passion qui n'a fait que grandir, le monde non-humain ou préhumain des bêtes des bois et des eaux, de la mer non polluée et des forêts non encore jetées bas ou défoliées par nous. En d'autres termes, que je prêtais à l'Empereur Hadrien lui-même, mon allégeance commençait à passer « du nageur à la vague ».

De cet élargissement de son souci du monde va prendre acte l'éventail des Sociétés auxquelles Yourcenar verse régulièrement des dons. ${ }^{19}$ Mais dans le domaine de l'activisme, il y a écologisme et écologisme. Celui, managérial, du développement durable et celui, éthique, de la symbiose harmonieuse. L'engagement de Marguerite Yourcenar est moins dans l'action directe d'un mouvement politique ou parapolitique, que dans un activisme indirect et personnel, à la fois associatif et américain par ses cotisations à diverses associations pour la défense de la nature et la lutte contre les pollutions, et intellectuel et français par ses discours et la création de la Fondation de Bailleuil. Car, depuis l'injonction de Pangloss à cultiver notre jardin, « il y a le plan local, il $\mathrm{y}$ a le plan individuel » qui nous invite à « prêcher d'exemple » (Sauver... 28).

Par sa critique radicale d'un anthropocentrisme mesquin et d'un humanisme idéaliste, par l'appel à ce « deuil de soi » que prônait Lévi-Strauss dans ses Tristes tropiques, Marguerite Yourcenar était annonciatrice de l'«écologie profonde» d'aujourd'hui, qui entend substituer aux valeurs humanistes -considérées immorales en ce qu'elles concèdent au reste du vivant le statut de « ressource »des valeurs suprahumanistes.

Avec des mots d'ordre tels que faire « machine arrière », «faire tache d'huile », faire baisser le surpeuplement humain, la pensée yourcenarien ne semble aspirer à la mise en œuvre d'un Projet de paix écologique perpétuelle, dont la mesure grecque et la bienveillance bouddhique seraient les moteurs, à seule fin de sauvegarder pour tous le « bel équilibre du monde » (Sauver..., 30).

\section{Bibliographie}

Bonali Fiquet, Françoise. «Yourcenar et la défense de l'environnement à travers les Entretiens ». Marguerite Yourcenar essayiste. Parcours, méthodes et finalités d'une écriture critique, Actes du colloque international de Modène, Parme et Bologne (5-8 mai 1999), volume dirigé par Carminella Biondi et al. SIEY, 2000, p. 45-54.

Bulletin du Cidmy. «Marguerite Yourcenar et l'écologie », n² 2, oct. 1990 [anthologie].

Chehab, May. «Presentación» à la partie "Discursos y conferencias». Marguerite Yourcenar y la ecología. Un combate ideológico y político, volume dirigé par Andrea Padilla et al. Universidad de los Andes, 2007, p. 69-71.

\footnotetext{
${ }_{19}$ Citons seulement leurs noms : Environmental Action, Friends of the Earth, The National Parks Conservation Association, The Natural Resources Council of Maine, le National Resources Defense Council, Nature Conservancy (dont certaines politiques ont été controversées, mais après la mort de Yourcenar), Save the Redwoods League, Scenic Hudson, Sierra Club, The Wilderness Society, Greenpeace USA, World Wildife Fund.
} 
Derrida, Jacques. L'animal autobiographique. Galilée, 1999.

. L'animal que donc je suis. Galilée, 2006.

Evano, Brigitte. "La sacralisation de la nature dans L'CEuvre au Noir ». Le sacré dans l'œuvre de Marguerite Yourcenar, Actes du colloque tenu à Bruxelles (26-28 mars 1992), volume dirigé par Rémy Poignault. SIEY, 1993, p. 211-218.

Fort, Pierre-Louis. "Appartenir au même règne : vies animales et humaines dans Mémoires d'Hadrien ». Lectures de Marguerite Yourcenar. Mémoires d'Hadrien, volume dirigé par Bruno Blanckeman. Presses Universitaires de Rennes, 2014, p. 199-214.

Kant, Emmanuel. Qu'est-ce que les lumières ?QEuvres philosophiques[1784], t. II. Traduit par François de Gandt, Gallimard, coll. «Bibliothèque de la Pléiade », 1985, p. 363-493.

Lévi-Strauss, Claude. Tristes Tropiques, Paris, Plon, 1955. NN$^{\circ} 5192$ de l'inventaire de la bibliothèque personnelle de Marguerite Yourcenar à Petite Plaisance.

Mallet, Marie-Louise (dir.). L'animal autobiographique, autour de Jacques Derrida. Galilée, 1999.

Schaeffer, Jean-Marie. La fin de l'exception humaine. Gallimard, 2007.

Torres Mariño, Vicente. «L'animal ou l'altérité sacrée chez Marguerite Yourcenar ». Les miroirs de l'altérité dans l'œuvre de Marguerite Yourcenar, Actes du colloque international de Bogota (10-11 mars 2011), volume dirigé par Rémy Poignault et Vicente Mariño Torres. SIEY, 2014, p. 199-210.

Vitalis, André et al. (dir.). Médias, temporalités et démocratie. Apogée/PUF, 2000.

Yourcenar, Marguerite. Théâtre I, Gallimard, coll. « Blanche », 1971.

" "Discours de Réception à l'Académie française», 22.1.1981, http://www.academie-francaise.fr/discours-de-reception-de-marguerite-yourcenar. . « L'Écologie » (en 3 parties). TV Canada, 1981.

la Pléiade », 1982. . OEuvres romanesques [OR], Gallimard, coll. «Bibliothèque de . «...Si nous voulons encore essayer de sauver la terre». Le droit à la qualité de l'environnement. Un droit en devenir, un droit à définir, Actes de la Ve Conférence internationale de droit constitutionnel, volume dirigé par Nicole Duplé. Éditions Québec/Amérique, 1988, p. 21-33.

Pléiade », 1991. . Essais et Mémoires [EM], Gallimard, coll. «Bibliothèque de la . Lettres à ses amis et quelques autres. Gallimard, 1995. . Portrait d'une voix. Gallimard, 2002. 